\title{
QUEEN'S
UNIVERSITY
BELFAST
}

\section{Short pulse laser-induced dissociation of vibrationally cold, trapped molecular ions}

Alexander, J., Calvert, C., King, R., Kelly, O., Bryan, T., Nemeth, G. R. A. J., Newell, W. R., Froud, C. A., Turcu, I. C. E., Springate, E., Orr, P. A., Pedregosa-Gutierrez, J., Walter, C. W., Williams, R. A., Williams, I., \& Greenwood, J. (2009). Short pulse laser-induced dissociation of vibrationally cold, trapped molecular ions. Journal of Physics B: Atomic, Molecular and Optical Physics, 42(15), [154027]. https://doi.org/10.1088/09534075/42/15/154027

Published in:

Journal of Physics B: Atomic, Molecular and Optical Physics

Document Version:

Publisher's PDF, also known as Version of record

Queen's University Belfast - Research Portal:

Link to publication record in Queen's University Belfast Research Portal

Publisher rights

(C) 2009 IOP Publishing

General rights

Copyright for the publications made accessible via the Queen's University Belfast Research Portal is retained by the author(s) and / or other copyright owners and it is a condition of accessing these publications that users recognise and abide by the legal requirements associated with these rights.

Take down policy

The Research Portal is Queen's institutional repository that provides access to Queen's research output. Every effort has been made to ensure that content in the Research Portal does not infringe any person's rights, or applicable UK laws. If you discover content in the Research Portal that you believe breaches copyright or violates any law, please contact openaccess@qub.ac.uk. 
Short pulse laser-induced dissociation of vibrationally cold, trapped molecular ions

This article has been downloaded from IOPscience. Please scroll down to see the full text article.

2009 J. Phys. B: At. Mol. Opt. Phys. 42154027

(http://iopscience.iop.org/0953-4075/42/15/154027)

View the table of contents for this issue, or go to the journal homepage for more

Download details:

IP Address: 143.117.13.202

The article was downloaded on 05/11/2010 at 14:59

Please note that terms and conditions apply. 


\title{
Short pulse laser-induced dissociation of vibrationally cold, trapped molecular ions
}

\author{
J D Alexander ${ }^{1}$, C R Calvert ${ }^{1}$, R B King ${ }^{1}$, O Kelly ${ }^{1}$, W A Bryan ${ }^{2}$, \\ G R A J Nemeth ${ }^{2}$, W R Newell ${ }^{3}$, C A Froud ${ }^{4}$, I C E Turcu ${ }^{4}$, E Springate ${ }^{4}$, \\ P A Orr ${ }^{5}$, J Pedregosa-Gutierrez ${ }^{6}, \mathrm{C}$ W Walter $^{7}$, R A Williams ${ }^{8}$, \\ I D Williams ${ }^{1}$ and J B Greenwood ${ }^{1}$
}

\footnotetext{
${ }^{1}$ School of Mathematics and Physics, Queen's University Belfast, Belfast BT7 1NN, UK

${ }^{2}$ Department of Physics, Swansea University, Swansea SA2 8PP, UK

${ }^{3}$ Department of Physics and Astronomy, University College London WC1E 6BT, UK

${ }^{4}$ Central Laser Facility, STFC Rutherford Appleton Lab, Didcot OX11 0QX, UK

${ }^{5}$ Northern Ireland Medical Physics Agency, Forster Green Hospital, Belfast BT8 6HD, UK

${ }^{6}$ Physique des Interactions Ioniques et Moleculaires, Université de Provence, 13397 Marseille, France

${ }^{7}$ Department of Physics and Astronomy, Denison University, Granville, OH 43023, USA

${ }^{8}$ Clarendon Laboratory, Oxford University, Oxford OX1 3PU, UK

E-mail: j.greenwood@qub.ac.uk
}

Received 28 January 2009, in final form 9 March 2009

Published 16 July 2009

Online at stacks.iop.org/JPhysB/42/154027

\begin{abstract}
An electrostatic trapping scheme for use in the study of light-induced dissociation of molecular ions is outlined. We present a detailed description of the electrostatic reflection storage device and specifically demonstrate its use in the preparation of a vibrationally cold ensemble of deuterium hydride $\left(\mathrm{HD}^{+}\right)$ions. By interacting an intense femtosecond laser with this target and detecting neutral fragmentation products, we are able to elucidate previously inaccessible dissociation dynamics for fundamental diatomics in intense laser fields. In this context, we present new results of intense field dissociation of $\mathrm{HD}^{+}$which are interpreted in terms of recent theoretical calculations.
\end{abstract}

(Some figures in this article are in colour only in the electronic version)

\section{Introduction}

If the properties of the most fundamental molecules, e.g. $\mathrm{H}_{2}^{+}$, $\mathrm{HeH}^{+}$and $\mathrm{H}_{3}^{+}$, are to be experimentally investigated, then techniques for creating these molecular ions with sufficient fluxes and densities are required. The limitation in performing such studies with ion beams is that the molecules are normally created in excited vibrational states which have radiative lifetimes much longer than the transit time from source to interaction region. With the development of storage rings for atomic and molecular physics, there have been significant advances in studies of molecular ions in welldefined states (Zajfman et al 2003, Wolf et al 2006). As the ions can be confined for many seconds, radiative relaxation or collisional quenching in an electron cooler produces molecular ions in their ground vibrational state, enabling unambiguous comparison between experiment and theory.
The recent development of compact storage rings and traps (Andersen et al 2004, Koelemeij et al 2007, Bernard et al 2008) now makes it possible for small research groups to create cold molecular ion beams for use in innovative new experiments. In this paper, we will demonstrate the use of a linear electrostatic ion trap in the preparation of a vibrationally cold $\mathrm{HD}^{+}$target for studies of light-induced dissociation. Since there are no magnetic or time-varying electric fields in our apparatus, the trapping scheme is mass independent and the method can be generally used to study the dissociation of simple or complex molecules by a pulsed light source.

An interesting area of research which could substantially benefit from these developments is the study of molecules in intense laser fields. In the past 10-15 years, there has been a concerted effort to study intense femtosecond pulse interactions with the $\mathrm{H}_{2}^{+}$molecule, and its isotopologues $\mathrm{HD}^{+}$ and $\mathrm{D}_{2}^{+}$. These 'simple' molecules have been studied in great 
detail, as an ideal test-bed for elucidating molecular dynamics in intense fields (see Posthumus 2004 and references therein). However, the response of the molecule to the laser field is strongly dependent on the vibrational state $v$, making it very difficult for experimental investigations to ascertain the basic physical mechanisms and energetics of the molecular breakup.

There are two main experimental schemes for preparing $\mathrm{H}_{2}^{+}$(or $\mathrm{HD}^{+} / \mathrm{D}_{2}^{+}$) targets for intense field studies, each creating different vibrational distributions. The most widely used approach is to interact an intense field pulse with a gas-phase $\mathrm{H}_{2}$ target, creating $\mathrm{H}_{2}^{+}$via tunnel ionization on the leading edge of the pulse. Due to a dependence of the ionization rate on the internuclear separation, this scheme has been found to predominantly populate lower vibrational levels and is extremely sensitive to both pulse intensity and wavelength (Saenz 2000, Urbain et al 2004). For an experimental ensemble of molecules it is therefore hard to characterize the exact vibrational distribution of a target created in this way. An alternative approach is to independently create a beam of hydrogenic ions and subsequently study their interaction with an intense laser. From the initial electron impact ionization in an ion source, a wide range of vibrational levels are populated in a Franck-Condon distribution. While this ion beam approach provides an $\mathrm{H}_{2}^{+}$(or $\mathrm{HD}^{+} / \mathrm{D}_{2}^{+}$) target with a well-characterized vibrational distribution, the resulting dissociation signals contain contributions from many vibrational levels which cannot be easily identified (Kiess et al 2008, McKenna et al 2007, Wang et al 2006, Pavičić et al 2005, Williams et al 2000).

In this context, we apply our trapping scheme to the preparation of a vibrationally cold target of $\mathrm{HD}^{+}$. We use $\mathrm{HD}^{+}$ rather than $\mathrm{H}_{2}^{+}$or $\mathrm{D}_{2}^{+}$as it has a permanent dipole moment and will radiatively cool into its ground vibrational state after approximately $300 \mathrm{~ms}$. As the ions are moving in the trap with $\mathrm{keV}$ energies, we are also able to study the pure dissociation of the molecular ion through emission of neutral particles, without the interference of any charged fragments produced from the ionization process. A brief report of this method has been previously communicated (Orr et al 2007), in which the above threshold dissociation of $\operatorname{HD}^{+}(v=0)$ was found to be the dominant dissociation process. This had not previously been observed experimentally as the ground state dissociation was overwhelmed by signal from excited states.

The paper is divided into the following sections. In section 2.1 we describe the ion trapping apparatus, while in section 2.2 we describe the laser interaction geometry and explain how the reaction products are analysed. We present new results of $\mathrm{HD}^{+}$dissociation in section 3 which are discussed in light of our previous results and recent theoretical calculations. In the conclusion we assess potential future developments and applications of the technique.

\section{Experimental techniques}

\subsection{Ion trap}

The ion trap has been briefly reported in an earlier publication (Orr et al 2007) but a more in-depth description is provided

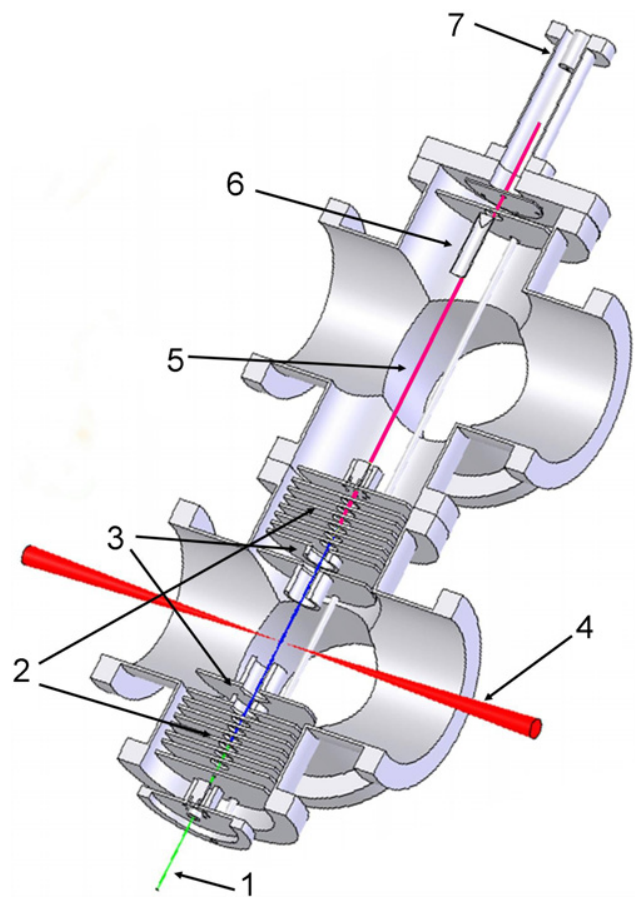

Figure 1. Schematic diagram of the ion trap, laser interaction and detection geometry. (1) Incident ion beam, (2) mirror electrodes, (3) lens electrodes, (4) fs laser beam, (5) neutral emission trajectories, (6) off-axis Faraday cup and (7) channel electron multiplier.

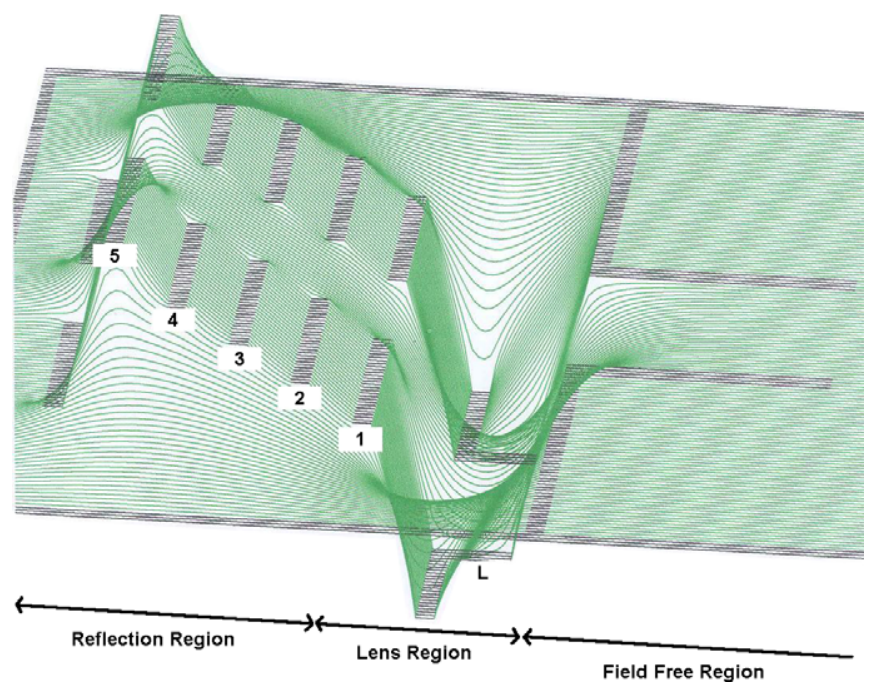

Figure 2. The potential energy surface generated by the ion trap electrode geometry, for reflection voltages $(1)=0 \mathrm{~V},(2)=$ $0.385 \mathrm{kV},(3)=0.75 \mathrm{kV},(4)=1.1 \mathrm{kV},(5)=1.75 \mathrm{kV}$ and a lens voltage $(L)=-2.0 \mathrm{kV}$.

here. The instrument operates in a manner analogous to a laser cavity (Pedersen et al 2002, Ota et al 2006), with electrostatic potentials and a linear geometry which preserves the $\mathrm{keV}$ beam characteristics of the injected ions. A cutaway of the device is shown in figure 1. It is based on the 'Weizmann trap' (Dahan et al 1998) and each end consists of a series of aperture plates, four of which possess increasing potentials so as to act as planar mirrors, with a lens providing the radial confinement (figure 2). The device has a total length of 
$330 \mathrm{~mm}$, with reflection, focusing and field-free regions of lengths approximately 50, 40 and $150 \mathrm{~mm}$, respectively. The actual distance the ions penetrate into the reflecting regions is dependent on the applied potentials but is typically $25-30 \mathrm{~mm}$. The reflecting plates are $3 \mathrm{~mm}$ thick, separated by $7 \mathrm{~mm}$ and have aperture diameters of $20 \mathrm{~mm}$. The lens has an inner diameter of $30 \mathrm{~mm}$.

Under the appropriate conditions, ions with positions and velocities close to the axis are stably confined as has been previously demonstrated using optical models, charged particle software simulations and experimental results (see, for example, Pedersen et al 2002, Ota et al 2006). Ion bunches are introduced into the trap by pulsing some (or all) of the potentials at one end to open and close the cavity.

The lifetime of the stored ions is limited by elastic and charge changing collisions with background gas and elastic collisions between the ions (Pedersen et al 2002). Neutralized ions are ejected and can be detected with an on-axis channel electron multiplier which provides a convenient measure of the ion population as a function of time. The geometry of our device differs from the original Weizmann device in that the total length has been reduced by $30 \%$, along with $25 \%$ larger apertures and lenses. While this reduces the ion bunch length which can be trapped, this shorter, fatter device has an increased angular acceptance, extending the stable phase space and reducing ion losses through scattering processes.

Another significant difference in the operation of our instrument is that we normally operate with a lens potential of opposite polarity to the ion charge. This accelerating lens requires larger potentials for stable trapping compared with a decelerating lens, but provides a larger stable phase space and thus more ions are accepted onto stable trajectories. Using the SIMION ${ }^{\circledR}$ software, stable trajectories were determined for ions launched parallel to the trap axis as a function of the initial distance from this axis. Singly charged positive ions with an energy of $1 \mathrm{keV}$ were simulated for mirror potentials 0.385 , $0.75,1.1,1.75 \mathrm{kV}$. These data, plotted in figure 3 as a function of lens potential, demonstrate three stable regions. Also shown is the phase space area occupied by 1000 simulated ions at the centre of the trap after more than a thousand oscillations. Once injected the ions quickly fill up this available stable phase space due to aberrations in the lenses and mirrors. It can be seen that negative lens potentials tend to have a larger stable phase space and are therefore more tolerant of the initial injection conditions compared with a positive lens. Qualitatively, this is due to a negative lens performing a sequence of converging, diverging, converging lens operations which keep the ion trajectories closer to the trap axis compared with a positive lens.

Figure 4 shows experimental neutral particle yields as a function of trapping time for positive $(+1.0 \mathrm{kV})$ and negative $(-3.6 \mathrm{kV})$ lens settings under similar conditions to those used for the simulations in figure 3. A $400 \mathrm{nA} 1 \mathrm{keV} \mathrm{H}_{2}^{+}$ion beam produced from a $10 \mathrm{GHz}$ permanent magnet electron cyclotron resonance (ECR) source was used to inject approximately $5 \times$ $10^{6}$ ions for each trap cycle. The trap was opened and closed by pulsing the two highest mirror potentials. For each lens setting, the injection conditions were varied to maximize the

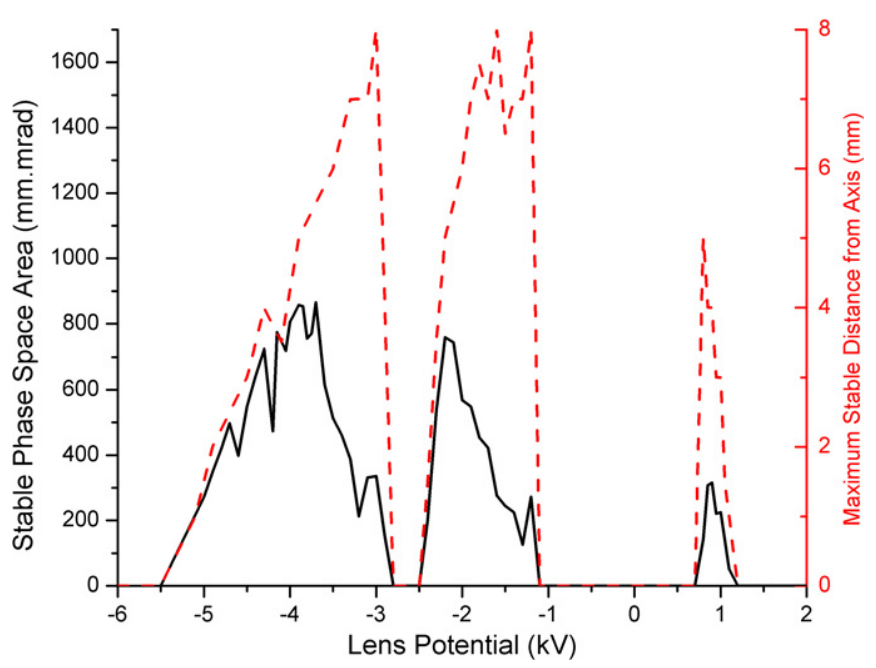

Figure 3. Stability of the ion trap as a function of lens potential plotted in terms of the maximum initial injection distance from the trap axis for which an ion can be stably trapped (dotted red curve), and the stable phase space area (solid black curve). See the text for the trap settings.

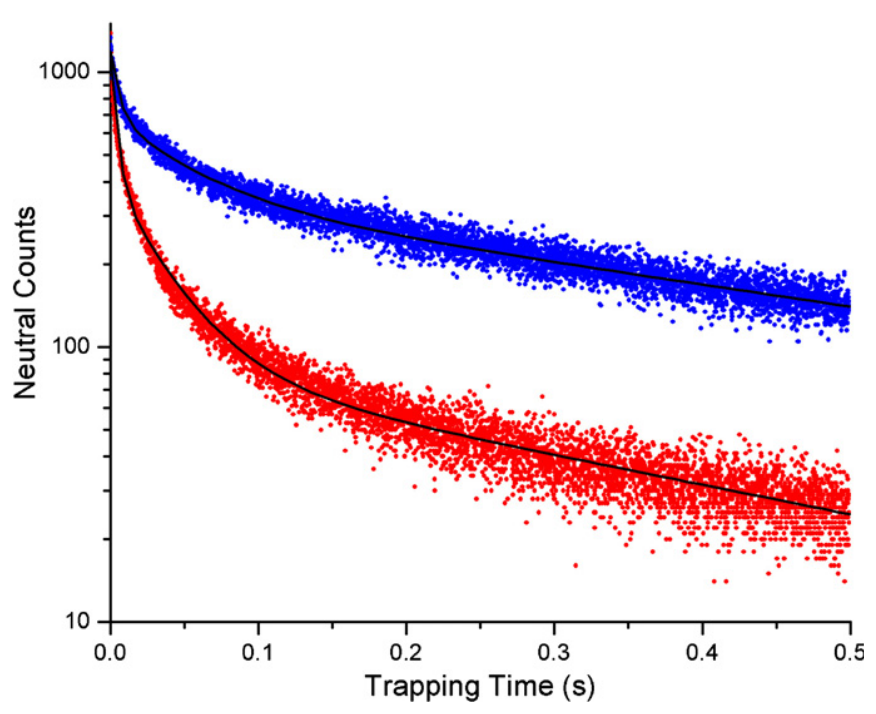

Figure 4. Survival of $\mathrm{H}_{2}^{+}$ions as a function of trapping time as measured by the yield of neutral particles obtained from collisions with the background gas, within $0.1 \mathrm{~ms}$ intervals, for lens potentials of $+1.0 \mathrm{kV}$ (red) and $-3.6 \mathrm{kV}$ (blue).

number of ions surviving for more than $100 \mathrm{~ms}$ at an operating pressure of just less than $10^{-9}$ mbar.

Third-order exponentials have been fitted to the data which yield lifetimes of approximately 4, 40 and $400 \mathrm{~ms}$ for both data sets. The shortest lifetime is predominantly determined by ions with unstable trajectories due to the initial injection conditions, while the longest lifetime is due to collisions with background gas (elastic and neutralizing). For short-to-intermediate trapping times, ion-ion collisions are also expected to be a significant loss mechanism. The relative ion loss for each decay component (short, medium, long) is $64 \%, 29 \%, 7 \%$ for the positive lens and $38 \%, 30 \%, 32 \%$ for the negative lens. This demonstrates that a higher proportion of the ions possess a long decay time for a negative lens. 


\subsection{Laser interaction and detection}

The laser pulse interacts with the trapped molecular ions in the field-free region of the apparatus (figure 1). For $\mathrm{HD}^{+}$the possible fragmentation channels are

$$
\begin{gathered}
\mathrm{HD}^{+} \rightarrow \mathrm{H}^{+}+\mathrm{D}, \\
\mathrm{HD}^{+} \rightarrow \mathrm{H}+\mathrm{D}^{+}, \\
\mathrm{HD}^{+} \rightarrow \mathrm{H}^{+}+\mathrm{D}^{+}+\mathrm{e}^{-} .
\end{gathered}
$$

Unlike neutral gas target experiments, detection of the neutral fragment is possible as the target molecule has a high kinetic energy. As a result, only the pure dissociation channels are observed, thus avoiding any interference from channel (3). Although the laboratory $\mathrm{keV}$ kinetic energies $E$ are much greater than the $\mathrm{eV}$ kinetic energy release in the centre-of-mass frame, a velocity transformation to the lab frame readily shows that it is possible to distinguish between different dissociation processes from the neutrals' time of flight $(\mathrm{ToF})$ to the channel electron multiplier (CEM).

In general, if the initial mass of the ion is $m$, the interaction produces neutral and ion fragments of masses $m_{1}$ and $(m-$ $m_{1}$ ) respectively, and $\Delta E$ of kinetic energy is shared between the two fragments; the dissociation velocity $u$ of the neutral fragment in the centre-of-mass frame is given by

$$
u=\left(\frac{2\left(m-m_{1}\right) \Delta E}{m_{1} m}\right)^{1 / 2} .
$$

Without any pre-alignment of the molecule or dynamical alignment during the interaction pulse, the molecular axis will be randomly orientated. If $\theta$ is the angle in the centre-of-mass frame between the trap axis and the molecular axis, $D$ is the distance between the interaction point and the CEM $(0.55 \mathrm{~m})$, and $v$ is the laboratory velocity of the molecular ions, the ToF to the detector is given by

$$
T=\frac{D}{v+u \cos \theta} .
$$

Due to the finite acceptance solid angle of the CEM, the values of $\theta$ which are accepted are limited to angles close to 0 and $180^{\circ}$. Therefore, if the polarization axis of the laser pulse is parallel to the trap axis, the observed signal is derived from molecules whose internuclear axis is aligned with the laser polarization. This is the geometry for which our results have been acquired as it provides the strongest dipole coupling between the ground and first excited electronic states. Alternatively, studies of the angular dependence of the dissociation can also be performed by successively rotating the polarization axis.

In the laboratory frame the half-angle acceptance of the CEM is $\phi \approx 7.5 \mathrm{mrad}$. As $u \ll v$ this gives the following condition for products to lie within the solid angle of the CEM:

$$
\sin \theta<\frac{v}{u} \phi
$$

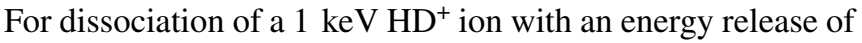
$\Delta E=1 \mathrm{eV}$, the orientation of the molecular axis of $\mathrm{HD}^{+}$with respect to the trap axis must be within $20^{\circ}$ for the D fragments to hit the CEM ( $10^{\circ}$ for $\mathrm{H}$ fragments). For each fragment this produces a forward and backward peak in the ToF spectrum,

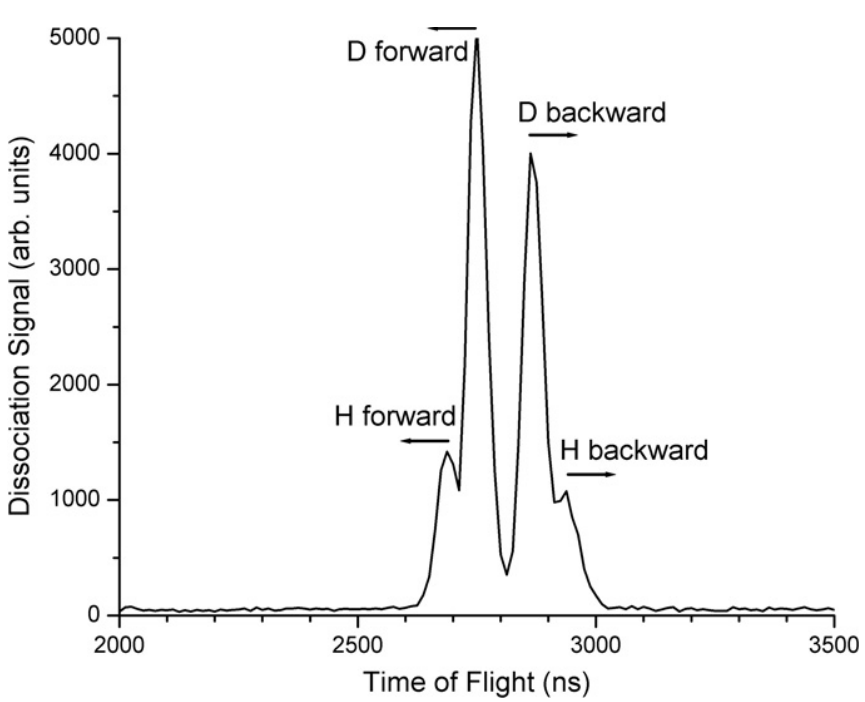

Figure 5. Typical time-of-flight spectrum obtained from dissociation of $1 \mathrm{keV} \mathrm{HD}^{+}$by low intensity $\left(10^{12} \mathrm{~W} \mathrm{~cm}^{-2}\right), 800 \mathrm{~nm}$, 40 fs laser pulses. The peaks are identified by the neutral fragments and their direction in the centre of mass corresponding to emission towards (forward) and away (backward) from the detector.

slightly spread by the $\cos \theta$ term. Taking the case of $\cos \theta=$ \pm 1 , the ToF is

$$
T \approx \frac{D}{v}\left(1 \pm\left(\frac{\left(m-m_{1}\right) \Delta E}{m_{1} E}\right)^{1 / 2}\right) .
$$

For dissociation of $\mathrm{HD}^{+}$with a single energy release process producing $\mathrm{H}$ and $\mathrm{D}$ fragments, this results in four distinct peaks as demonstrated by the data in figure 5. The two large peaks are from D neutrals while the smaller shoulders are from $\mathrm{H}$ neutrals. The peak separations are due to a $0.8 \mathrm{eV}$ energy release from absorption of one $800 \mathrm{~nm}$ photon $(1.55 \mathrm{eV})$ by vibrational levels around $v=10$. Detection of $\mathrm{H}$ fragments is suppressed as they have half the laboratory energy of the D fragments resulting in a lower detection efficiency and a lower centre-of-mass solid angle of acceptance.

The peak widths in the spectrum are predominantly due to the energy spread $\delta E$ of the ions produced from the ion source. In the present study, the energy spread for a $1 \mathrm{keV} \mathrm{HD}^{+}$beam was about $\delta E=30 \mathrm{eV}$, introducing a width in the ToF peak of

$$
\delta T \approx \frac{D}{v} \frac{\delta E}{2 E}=33 \mathrm{~ns} .
$$

The yield of laser-produced neutrals was used to optimize the vertical positioning of the laser with respect to the trapped ions. By measuring this yield as a function of the laser height $(y)$, the diameter of the trapped ion beam was estimated to be $2 \mathrm{~mm}$ full width half maximum. For the conditions used in figure 4 with a negative lens potential, this corresponds to an ion target density of around $10^{6} \mathrm{~cm}^{-3}$ after $100 \mathrm{~ms}$ of confinement.

\section{Results and discussion}

$\mathrm{H}_{2}^{+}$and its isotopologues are not only the most fundamental molecules available for the study of strong field dissociation processes, but they provide an almost pure two electronic state 


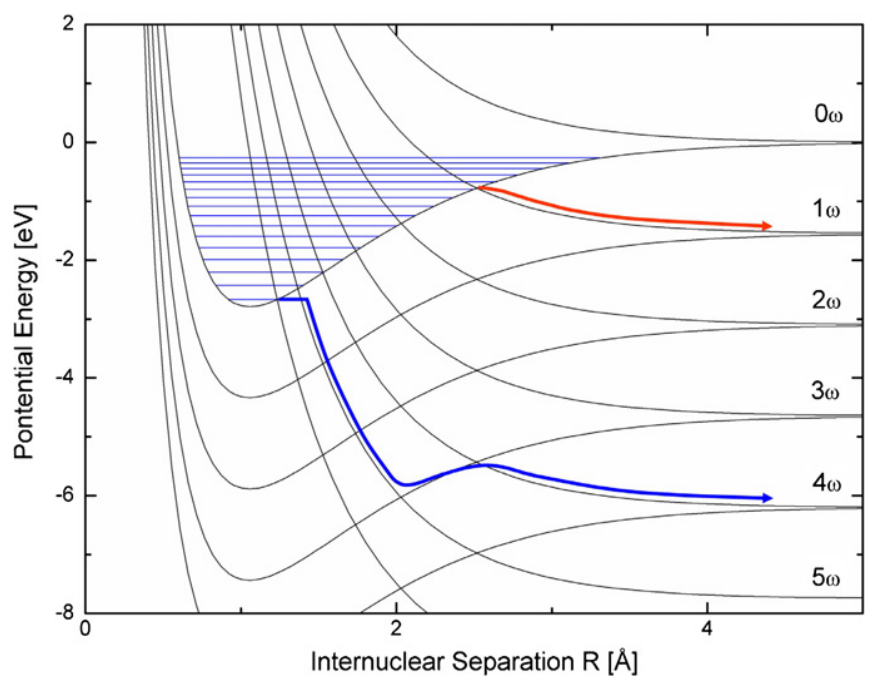

Figure 6. $\mathrm{HD}^{+}$adiabatic potential energy curves dressed with an integer number of $800 \mathrm{~nm}$ photons. Possible dissociation paths are sketched for vibrational states $v=10$ (red arrow) and $v=0$ (blue arrow).

system since the $1 \mathrm{~s} \sigma_{g}$ ground state and $2 \mathrm{p} \sigma_{u}$ first excited state are well separated in energy from other excited states. The interaction with an intense light field is often considered in terms of the photon-dressed potentials, where solutions of the light field-molecule Hamiltonian in the Floquet approach (Chu 1981) yield a series of potential energy curves each separated by one $800 \mathrm{~nm}$ photon (see figure 6). As the field strength increases, the off-diagonal elements in the Floquet matrix (which are due to dipole coupling between states) introduce adiabatic avoided curve crossings. For the homonuclear $\mathrm{H}_{2}^{+}$and $\mathrm{D}_{2}^{+}$molecules, coupling only occurs between curves differing in odd numbers of dressed photons due to parity considerations. However, for heteronuclear $\mathrm{HD}^{+}$, coupling via absorption of an even number of photons is also possible (Kiess et al 2008).

This coupling between dressed potential energy curves gives a useful physical picture of how strong field dissociation proceeds. For instance, $\mathrm{HD}^{+}$molecules populated in vibrational state $v=10$ reside at a photon crossing with the one-photon-dressed $2 \mathrm{p} \sigma_{u}$ state. Even in weak fields this coupling is strong enough to open up an avoided crossing through which the molecule can dissociate resulting in the release of about $0.8 \mathrm{eV}$ of kinetic energy (figure 5, red arrow in figure 6). Lower-lying vibrational states may also dissociate through this channel if they tunnel through the light-induced potential barrier. For more tightly bound vibrational states, only higher-order couplings at curve crossings can result in dissociation. For the ground state $v=0$ this involves coupling at a five or six photon crossing followed by possible re-emission or absorption of more photons as the molecule dissociates. The blue curve in figure 6 gives a sketch of one possible path which involves absorption of five photons, followed by emission of two onto a three photon curve, and absorption of a further photon at another crossing resulting in net absorption of four photons.

This picture highlights the difficulty of experimentally identifying the mechanisms contributing to dissociation, where

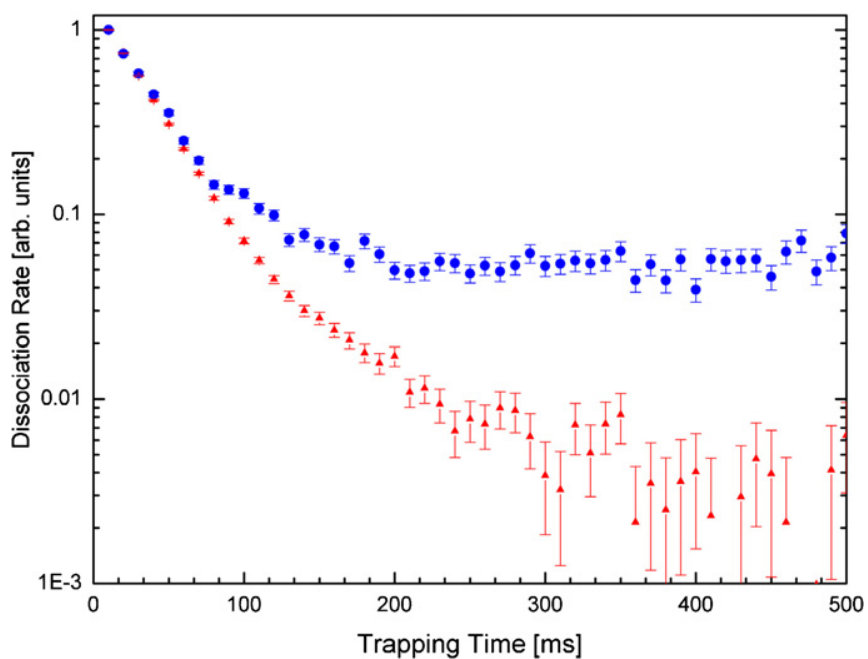

Figure 7. Relative dissociation rate of $\mathrm{HD}^{+}$ions for a 40 fs pulse as a function of trapping time. Blue circles: on focus interaction with peak intensity of $10^{15} \mathrm{~W} \mathrm{~cm}^{-2}$. Red triangles: off-focus interaction at $5 \times 10^{12} \mathrm{~W} \mathrm{~cm}^{-2}$. Note the two data sets have been normalized to the signal rate obtained at zero time.

different vibrational states will respond differently to the laser field. By introducing the present trapping techniques to this field of research, we have been able to perform the first measurements on hydrogen molecular ions populated only in their ground vibrational state.

The results presented in this paper were obtained from two different Ti:Sapphire lasers utilized at the Astra facility of the Rutherford Appleton Laboratory. Both of these lasers operated at a central wavelength of $800 \mathrm{~nm}$, with a repetition rate of $1 \mathrm{kHz}$ and were focussed by a $25 \mathrm{~cm}$ focal length lens. One laser system generated $0.5 \mathrm{~mJ}$ in a $40 \mathrm{fs}$ pulse producing a maximum focussed intensity of around $10^{15} \mathrm{~W} \mathrm{~cm}^{-2}$ (hereafter referred to as laser 1), while the second generated $4 \mathrm{~mJ}$ in $30 \mathrm{fs}$ and a maximum intensity of $10^{16} \mathrm{~W} \mathrm{~cm}^{-2}$ (laser 2).

Some of the results for the dissociation of ground state $\mathrm{HD}^{+}$using laser 1 have already been presented in a previous publication (Orr et al 2007), in which above threshold dissociation (ATD) was found to be the dominant dissociation process. Despite the fact that two photons are the minimum required for dissociation, absorption of at least four photons had the highest probability (for a possible path see figure 6).

While these results focussed exclusively on the ground vibrational state, the technique also enables the fragmentation signal to be acquired as a function of trapping time, thus allowing different vibrational populations to be sampled. Assuming the $\mathrm{HD}^{+}$ions are created in the ion source with a Franck-Condon distribution of vibrational populations, table 1 provides the population distribution as a function of the trapping time. The dissociation signal after a particular trapping time can be acquired by integrating the ToF signal (e.g. figure 5) and dividing it by the fraction of ions remaining (from figure 4).

Figure 7 shows the change in dissociation rate as a function of trapping time for two different interaction intensities. It can be seen that the dissociation rate 
Table 1. The vibrational population of $\mathrm{HD}^{+}$as a function of trapping time assuming an initial Franck-Condon distribution (Amitay 1999), calculated using theoretical vibrational lifetimes (Amitay 1994).

\begin{tabular}{|c|c|c|c|c|c|c|c|c|c|c|c|c|}
\hline \multirow{2}{*}{$\begin{array}{l}\text { Trapping } \\
\text { time (ms) }\end{array}$} & \multicolumn{12}{|c|}{ Vibrational level $v$ population $(\%)$} \\
\hline & 0 & 1 & 2 & 3 & 4 & 5 & 6 & 7 & 8 & 9 & 10 & 11 \\
\hline 0 & 6.37 & 13.14 & 16.02 & 15.40 & 13.04 & 10.27 & 7.70 & 5.54 & 4.11 & 4.83 & 2.05 & 1.54 \\
\hline 10 & 8.83 & 15.93 & 17.48 & 15.39 & 12.36 & 9.03 & 6.68 & 5.02 & 3.84 & 3.18 & 1.52 & 0.74 \\
\hline 20 & 11.76 & 18.63 & 18.47 & 15.03 & 11.39 & 7.92 & 5.88 & 4.40 & 3.05 & 2.13 & 0.99 & 0.36 \\
\hline 40 & 18.90 & 23.32 & 19.09 & 13.44 & 9.22 & 6.03 & 4.28 & 2.84 & 1.57 & 0.88 & 0.34 & 0.08 \\
\hline 70 & 31.98 & 27.48 & 17.24 & 10.06 & 6.02 & 3.43 & 2.04 & 1.06 & 0.44 & 0.20 & 0.06 & 0.01 \\
\hline 100 & 46.19 & 27.77 & 13.51 & 6.52 & 3.29 & 1.54 & 0.74 & 0.30 & 0.10 & 0.04 & 0.01 & 0.00 \\
\hline 150 & 67.68 & 22.02 & 6.84 & 2.28 & 0.81 & 0.27 & 0.09 & 0.03 & 0.01 & 0.00 & 0.00 & 0.00 \\
\hline 200 & 82.84 & 13.83 & 2.59 & 0.56 & 0.13 & 0.03 & 0.01 & 0.00 & 0.00 & 0.00 & 0.00 & 0.00 \\
\hline 250 & 91.66 & 7.42 & 0.79 & 0.11 & 0.02 & 0.00 & 0.00 & 0.00 & 0.00 & 0.00 & 0.00 & 0.00 \\
\hline 300 & 96.17 & 3.60 & 0.21 & 0.02 & 0.00 & 0.00 & 0.00 & 0.00 & 0.00 & 0.00 & 0.00 & 0.00 \\
\hline 400 & 99.26 & 0.73 & 0.01 & 0.00 & 0.00 & 0.00 & 0.00 & 0.00 & 0.00 & 0.00 & 0.00 & 0.00 \\
\hline 500 & 99.87 & 0.13 & 0.00 & 0.00 & 0.00 & 0.00 & 0.00 & 0.00 & 0.00 & 0.00 & 0.00 & 0.00 \\
\hline
\end{tabular}

initially drops dramatically as higher vibrational levels, which readily dissociate by one-photon absorption, are radiatively depopulated. After $100 \mathrm{~ms}$, these levels are empty and dissociation of lower levels by higher-order crossings is no longer swamped by the signal from one photon dissociation. These lower-lying vibrational states have longer radiative lifetimes, so the signal subsequently drops more slowly from 100 to $200 \mathrm{~ms}$. After $300 \mathrm{~ms}$, it can be seen that at both intensities the dissociation rate is constant as would be expected for a steady vibrational population.

Our previous study (Orr et al 2007) showed that the more tightly bound $v=0$ state was difficult to dissociate resulting in very low signal rates. We have recently reduced the operating trap pressure to low $10^{-10}$ mbar and by using a negative lens setting (see section 2.1) we have increased the density of our $\mathrm{HD}^{+}(v=0)$ target by a factor of 5 . These improvements coupled with the higher specifications of laser 2 dramatically improved the signal rate. In figure 8(a) a series of ToF spectra are displayed for trapping times between (a) 0-20 ms and (b) 300-1100 ms using laser 2. Each successive spectrum corresponds to a shift of the focusing lens by $1 \mathrm{~mm}$ along the laser propagation direction $(z)$ allowing different regions of the laser focus to interact with the ions. This $z$-scan technique employed in some of our previous work (McKenna et al 2007) is an effective means of reducing the intensity while maintaining good signal yield through the increased overlap volume. At $z=0$, a peak intensity of $10^{16} \mathrm{~W} \mathrm{~cm}^{-2}$ was determined from the charge state yield of Xe gas ionized in a separate apparatus with the same focusing geometry (Bryan et al 2006).

Translation of the lens to $z=3 \mathrm{~mm}$ and $z=10 \mathrm{~mm}$ corresponds to a reduction of the interaction intensity to approximately $10^{15}$ and $10^{14} \mathrm{~W} \mathrm{~cm}^{-2}$ respectively. In figure 8(a), the separation of the main $D$ product peaks corresponds to an energy release of about $\Delta E=0.6 \mathrm{eV}$, which is due to dissociation of vibrational states just below the onephoton crossing. As the lens is translated, the ToF spectrum remains unchanged and there is a dip in the signal rate at the laser focus. Since a one-photon process is linear with intensity, the signal rate simply scales with the interaction volume subtended by the laser focus and the ion beam target. In
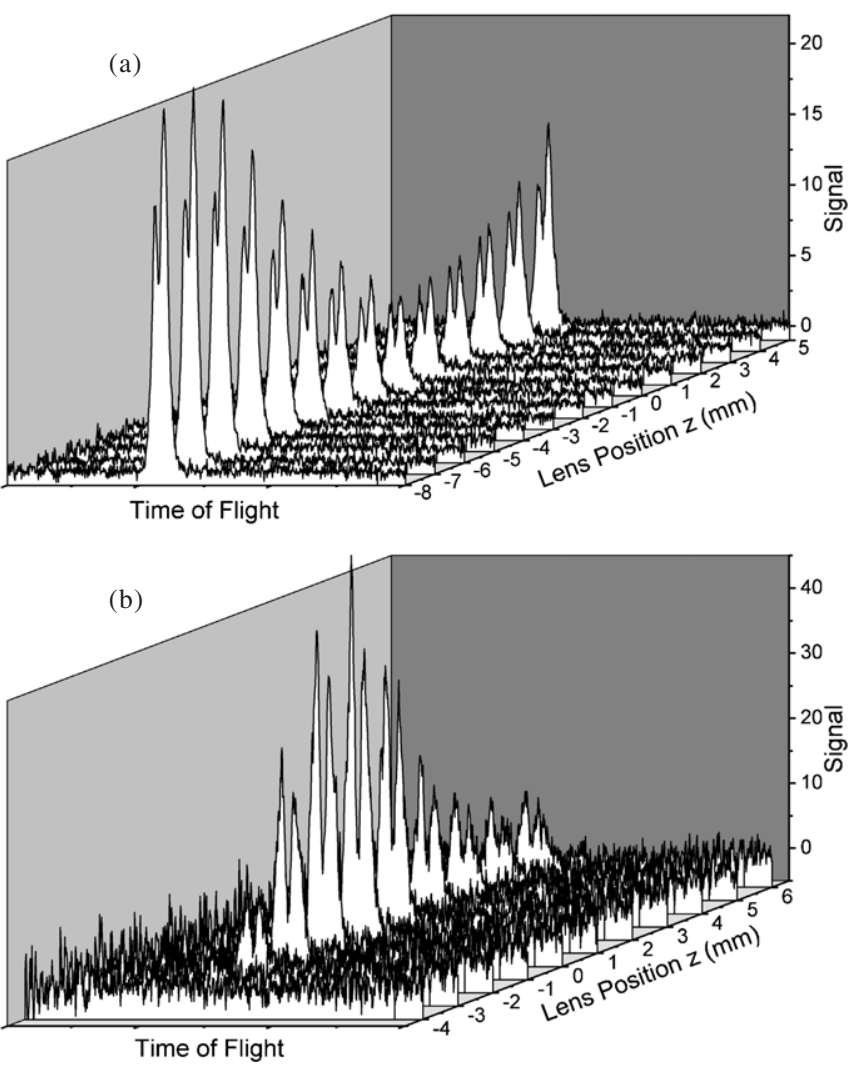

Figure 8. Time-of-flight spectra of $\mathrm{HD}^{+}$dissociation using laser 2 as a function of the focusing lens position for trapping times between: (a) 0-20 ms; (b) 300-1100 ms. $z=0$ corresponds to the laser focus interacting with the centre of the ion beam which possesses a full width half maximum of about $2 \mathrm{~mm}$. At focus, the laser waist was approximately $35 \mu \mathrm{m}$.

contrast to this, figure $8(\mathrm{~b})$ shows the $\operatorname{HD}^{+}(v=0)$ dissociation signal sharply peaked around the laser focus indicating the strong nonlinear nature of the process. In this case, the energy release corresponds to $\Delta E=0.9 \mathrm{eV}$ which is considerably lower than our previous results using laser 1 where dissociation predominantly occurred by the ATD mechanism with $\Delta E \approx$ $4 \mathrm{eV}$. For $z>3 \mathrm{~mm}$ where intensities are equivalent to the laser 1 results, there is still no evidence for this ATD process with laser 2. 
This inconsistency can only be explained by differences in the pulse lengths of each laser employed. While one might not expect a reduction from 40 to 30 fs to have such a strong influence, this conclusion is supported by a recent theoretical study which mirrors our findings (Song-Yuc et al 2008). In this paper, the kinetic energy release for dissociation of $\mathrm{HD}^{+}$ $(v=0)$ at an intensity of $6 \times 10^{14} \mathrm{~W} \mathrm{~cm}^{-2}$ and a wavelength of $800 \mathrm{~nm}$ was determined for pulse lengths of 10, 30, 40 and $60 \mathrm{fs}$. At 10 and $30 \mathrm{fs}$ they found that two and three photon absorption dominated while at 40 and 60 fs four photon absorption was most likely.

For a short pulse, the more rapid reduction in field strength as the molecule dissociates reduces the adiabatic coupling at subsequent curve crossings, reducing the absorption or reemission of photons. This has been demonstrated in previous studies (Peng et al 2005, McKenna et al 2008), but only for pulse lengths less than 10 fs. The ATD peaks are also shifted down in energy due to the AC Stark shift of the potential well. However, for this average potential to be physically meaningful, the field amplitude must remain constant for a number of cycles. It could be that in moving to use of a shorter pulse, we have observed a transition from an adiabatic to non-adiabatic regime.

\section{Conclusions}

We have constructed a tabletop linear ion trap, which can be used to confine beams of molecular ions for several seconds, during which time radiative decay from excited vibrational levels produces vibrationally cold molecules. It has been employed for the first experimental studies on the intense laserinduced dissociation of the fundamental $\mathrm{HD}^{+}$molecule in its ground vibrational state. By preparing such a target in a welldefined quantum state, the present work in combination with our previous results has unequivocally shown the existence of an above threshold dissociation mechanism and demonstrated a dramatic transition in the number of absorbed photons as the pulse length is shortened from 40 to $30 \mathrm{fs}$. Since our results are free of any interference from contributions by higher vibrational levels, we have been able to make direct comparison with recent theoretical results which supported our findings.

This technique provides a means for studying quantum control of the $\mathrm{HD}^{+}$reaction channels (1) and (2). It has been demonstrated in an experiment using a $\mathrm{D}_{2}$ gas target that the final destination of the electron in the dissociation of $\mathrm{D}_{2}^{+}$can, to a degree, be controlled by the carrier-envelope phase of an ultrashort laser pulse (Kling et al 2006). There are a number of theoretical predictions which show that more significant localization can be achieved using $\mathrm{HD}^{+}$, when it is populated in its ground vibrational state (Roudnev and Esry 2007, He et al 2007). We are now in a position to perform such experiments using ultrashort pulses and to investigate other fundamental molecules.

Moreover, it is important to highlight that the application of our trapping apparatus is not limited to the studies reported here. For instance, developments in free electron laser technology now open up previously inaccessible transitions in
$\mathrm{HeH}^{+}$(Pedersen et al 2007). The use of electrostatic potentials in our instrument enables large molecules such as DNA to be trapped, whose stability under exposure to various types of radiation could be studied. It can also be used as a highresolution mass spectrometer by detecting the image charge of ion bunches using a pick-up ring, since the oscillation frequency is inversely proportional to the square root of the mass (Strasser et al 2002, Bhushan et al 2007).

We note that such studies could be further enhanced by modifications to the current set-up. These might include: installing a position sensitive detector to image the reaction products (Pavičić et al 2005, Wang et al 2006); studying ionic fragments by pulsing the trap potentials allowing them to remain trapped (Stochkel et al 2008) or ejecting them using a trap with a bent geometry (Aviv et al 2008); improving the ToF resolution by reducing the energy spread in the ions, either directly from the ion source or by phase space manipulation of the ions while trapped (Goldberg et al 2003).

In conclusion, we have demonstrated that our experimental arrangement can open up new physics in the interaction of intense laser pulses with molecular ions. The apparatus is relatively simple to construct and control, and is compact enough to be transportable to major facilities.

\section{Acknowledgments}

This work was in part supported by the European Commission's ITSLEIF integrated infrastructure initiative. $\mathrm{CRC}$ and RBK have been supported by Department of Employment and Learning, Northern Ireland, JDA by the European Social Fund, OK by the Leverhulme Trust, PAO by Higher Education Authority, Ireland, JPG by Queen's University Belfast, RAW by the Nuffield Foundation and CWW by the Distinguished Visiting Fellowship program of the International Research Centre for Experimental Physics at Queen's University Belfast.

\section{References}

Amitay Z et al 1999 Phys. Rev. A 603769

Amitay Z, Zajfman D and Forck P 1994 Phys. Rev. A 502304

Andersen L H, Heber O and Zajfman D 2004 J. Phys. B 37 R57

Aviv O, Toker Y, Errit M, Bhushan K G, Pedersen H B, Rappaport M L, Heber O, Schwalm D and Zajfman D 2008 Rev. Sci. Instrum. 79083110

Bernard J, Montagne G, Bredy R, Terpend-Ordaciere B, Bourgey A, Kerleroux M, Chen L, Schmidt H T, Cederquist H and Martin S 2008 Rev. Sci. Instrum. 79075109

Bhushan K G, Gadkari S C, Yakhmi J V and Sahni V C 2007 Rev. Sci. Instrum. 78083302

Bryan W A et al 2006 Nature Phys. 2379

Chu S I 1981 J. Chem. Phys. 752215

Dahan M, Fishman R, Heber O, Rappaport M, Altstein N, Zajfman D and Van Der Zande W J 1998 Rev. Sci. Instrum. 6976

Goldberg S, Strasser D, Heber O, Rappaport M L, Diner A and Zajfman D 2003 Phys. Rev. A $6 \mathbf{8} 043410$

He F, Ruiz C and Becker A 2007 Phys. Rev. Lett. 99083002

Kiess A, Pavicic D, Hansch T W and Figger H 2008 Phys. Rev. A 77053401

Kling M F et al 2006 Science 312246 
Koelemeij J C J, Roth B, Wicht A, Ernsting I and Schiller S 2007 Phys. Rev. Lett. 98173002

McKenna J et al 2007 J. Phys. B 402607

McKenna J et al 2008 Phys. Rev. Lett. 100133001

Orr P A, Williams I D, Greenwood J B, Turcu I C E, Bryan W A, Pedregosa-Gutierrez J and Walter C W 2007 Phys. Rev. Lett. 98163001

Ota T, Saito M, Yokota A and Haruyama Y 2006 Japan J. Appl. Phys. 455263

Pavičić D, Hansch T W and Figger H 2005 Phys. Rev. A 72053413

Pedersen H B et al 2007 Phys. Rev. Lett. 98223202

Pedersen H B, Strasser D, Heber O, Rappaport M L and Zajfman D 2002 Phys. Rev. A 65042703

Peng L Y, Williams I D and McCann J F 2005 J. Phys. B 381727

Posthumus J H 2004 Rep. Prog. Phys. 67623

Roudnev V and Esry B D 2007 Phys. Rev. A 76023403
Saenz A 2000 J. Phys. B 334365

Song-Yuc B A, Kai-Jun Y, Young-Chang H and Shu-Lin C 2008 Chin. Phys. Lett. 252845

Stochkel K et al 2008 Rev. Sci. Instrum. 79023107

Strasser D et al 2002 Phys. Rev. Lett. 89283204

Urbain X, Fabre B, Staicu-Casagrande E M, de Ruette N, Andrianarijaona V M, Jureta J, Posthumus J H, Saenz A, Baldit E and Cornaggia C 2004 Phys. Rev. Lett. 16163004

Wang P Q, Sayler A M, Carnes K D, Xia J F, Smith M A, Esry B D and Ben-Itzhak I 2006 Phys. Rev. A 74043411

Williams I D et al 2000 J. Phys. B 332743

Wolf A, Buhr H, Grieser M, von Hahn R, Lestinsky M, Lindroth E, Orlov D A, Schippers S and Schneider I F 2006 Hyperfine Interact 172111

Zajfman D, Schwalm D and Wolf A 2003 Hyperfine Interact 146265 